a favourite example for such study and it has been used by Dr J. G. Atabekov (Moscow State University). Dr Atabekov-who was unable to be present at the meeting--has isolated fragments of the viral RNA and has found that fragments from the $5^{\prime}$ hydroxyl end have a greatly enhanced ability to reassemble with the protein. These results confirm the earlier work of P. J. G. Butler and A. Klug (MRC Laboratory of Molecular Biology, Cambridge).

A more complex system, and one where such detailed recognition of the viral RNA does not seem to occur, is found in cowpea chlorotic mottle and brome mosaic viruses. The RNA of these viruses occurs in three physically distinct but very similar types of particle, each of which is encapsidated with the same viral coat protein. Using this natural cleavage of the viral genome, Dr J. B. Bancroft (John Innes Institute) has obtained a hybrid virus, with part of its genome derived from each of the parent viruses. He has also been isolating mutants of some of the viral functions and, by the use of such mixed genome viruses, he hopes to map these mutations in spite of the lack of in vivo complementation which has proved so fruitful for such studies on bacteriophage.

A surprising result was reported by Dr A. M. Glauert (Strangeways Research Laboratory, Cambridge) who has investigated a protein component on the outer surface membrane of Gramnegative bacteria. After either freeze etching or negative staining these protein subunits are seen in the electron microscope to form a regular lattice which covers the maximum possible area of the surface. The subunits are released from the surface membrane, or even directly from the cell wall, by sonication and they will then reassemble to form a lattice which is very similar to the original one on the cell surface. This reassembly appears to take place at the air/water interface of a drop of the solution and without requiring any template or other information which is not within the protein subunits themselves. It is thus an example of a selfassembling system, and the main interaction with the other components of the membrane is presumably to require them as a surface upon which to assemble.

A more complicated assembly process occurs in the formation of bacterial flagella, where the rapid repolymerization of isolated flagellin, under mild conditions, has previously been shown by S. Asakura (Nagoya University, Japan) to require seed fragments derived from flagella filaments. Dr D. Kerridge (University of Cambridge) reviewed the structure of the bacterial flagellum and described how the filament is attached to the cell through a "hook" region and a basal body and growth occurs only at the distal end of the filament. $\mathrm{He}$ also showed that the nature of the filament is determined by the protein from which it is assembled, with some mutant proteins forming only straight filaments, even when polymerized onto the ends of seeds from the normal helical filaments. The detailed structure of the filament was described by Dr A. Klug and there followed a discussion of how the transfer of energy from the base along the length of the filament, for the beating of the flagellum, could be an inherent property of the structure and not require any physical transport to accompany it.

The assembly of bacterial ribosomes (S. Fahnestock, University of Wisconsin) and of bacteriophage (W. B. Wood, California Institute of Technology, and E. Kellenberger, Mikrobiologie Biozentrum, Basle) have each filled many whole meetings and were only discussed very briefly. The possibility of in vitro complementation for defective mutants in the assembly of bacteriophage T4 has provided much knowledge of the sequence of events in this process and there are the interesting demonstrations of proteins which are not incorporated into the final virus (for example, the product of gene 38 ) or which are cleaved during the maturation process (for example, the product of gene 23).

For an organelle as complex and autonomous as the mitochondrion, the prospect of understanding the assembly is still distant. Dr B. Afzelius (University of Stockholm) described the structures which have been observed during mitochondrial growth and duplication and showed how these events have similarities to cell replication. Taking advantage of these processes to study DNA synthesis, Dr A. C. Arnberg (University of Gröningen and Wallen- berg Laboratory, Uppsala) has shown the generation of a "D loop" by the synthesis of a single DNA strand, of about 500 nucleotides, complementary to one of the parental strands. At this point synthesis is checked and many of these structures are found in the DNA from a normal population of mitochondria. When DNA replication occurs, the synthesis of the same single strand resumes and synthesis of the complementary strand does not start until about 60 per cent of the DNA length has been copied. This second strand is then synthesized along the displaced parental strand, leading to the familiar semi-conservative replication.

\section{NUTRITION}

\section{Diet and Heart Disense}

from a Correspondent

CaRdiovascular disease is almost certainly of multifactorial aetiology, and the Nutrition Society held a symposium on July 5 and 6 at the University of Surrey on the subject of diet as a risk factor in the disease.

Dr A. G. Shaper (London School of Hygiene and Tropical Medicine) drew attention to the finding between different countries that the severity of atherosclerosis seems to be related to serum cholesterol concentration, and to the high proportions of dietary energy derived from fat, but that is not necessarily true for individuals within any one country. Epidemiological studies suggest that hypertension is almost as powerful as the serum cholesterol concentration as a predictor of susceptibility to heart disease. Scme workers had suggested that an increased concentration of serum triglyceride, independent of cholesterol, is also involved,

\title{
A Choice of Disulphide Pairings
}

IN next Wednesday's Nature New Biology (August 2) McKenzie and Shaw describe a situation in which two alternative disulphide pairings exist in a single protein molecule. In bovine $\beta$-lactoglobulin B, which is made up of a single species of chain, containing 162 residues, there is a single thiol group and two disulphide bonds per subunit. The thiol occurs in a part of the chain that contains two half-cystines (at positions 68 and 70) in a sequence ala-cys-glu-cysleu. One of these is implicated in a disulphide bridge with cys-57 at the other end. Thiol labelling with radioactive iodoacetamide proceeds satisfactorily only in $8 \mathrm{M}$ urea.

Subsequent peptide mapping reveals that the total counts taken up are distributed equally between two residues identified as cys-68 and cys-70. The same result was obtained for other genetic forms of the protein. The question then is whether the two alternative disulphide pairings pre-exist in the molecule, or are the result of disulphide exchange during the period of some minutes in which it is exposed to the $8 \mathrm{M}$ urea. The first alternative seems much the more probable, for the labelling ratio of the two positions was unaffected by the length of reaction or changes in the reaction $p \mathrm{H}$, which would be expected to alter the extent of any exchange process.

It therefore seems that here is a case in which two disulphide pairings have comparable energetic probabilities. McKenzie and Shaw point out that such a situation could easily be overlooked in other proteins if specific experimental precautions are not taken. 